\title{
Cryo-EM of Mitochondria: A Tale of Two Membranes
}

\author{
C.A. Mannella, C. Hsieh, C. Renken, D. Mankus, M. Marko
}

Resource for Visualization of Biological Complexity, Wadsworth Center, Albany, NY 12201-0509

It is the best of organelles, it is the worst of organelles. The mitochondrion generates the ATP that powers the cell's nanomachinery, while playing an integral role in numerous metabolic and signaling pathways essential to cell function. However, this organelle is also the main source of reactive oxygen species which, over time, can damage mitochondrial DNA and initiate a downward spiral of mitochondrial function that has been implicated in aging and a wide range of degenerative diseases [1].

Conventional transmission electron microscopy (EM) provided the first insights into the internal architecture of this organelle, showing an outer membrane (OM) surrounding a highly convoluted inner membrane (IM) that, in turn, encloses a crowded matrix space. Electron tomography has provided dramatic imagery of the complex topology of the energy-transducing IM [2] and, combined with techniques like cryo-sectioning [3] and cryo-FIB milling [4], offers the promise of revealing the in situ molecular organization of mitochondrial intra- and inter-membrane complexes, as well as that of the macromolecular assemblies (such as ribosomes) operating in the matrix.

Two examples of how EM is helping to resolve fundamental controversies related to phenomena at the mitochondrial surface, its interface with the rest of the cell, are discussed here: (1) linking the native structure of the channel in the mitochondrial OM (VDAC) to atomic structures of the channel obtained by x-ray crystallography and NMR, and (2) revealing the nature of contact sites between the OM and IM.

\section{Validation of the atomic structures of the VDAC pore.}

Three laboratories have independently determined the atomic structure of the mitochondrial outer membrane channel, VDAC (Voltage-Dependent, Anion-selective Channel), applying multidimensional NMR and x-ray crystallography to recombinant mammalian proteins expressed in bacteria and suspended in detergent micelles or lipid bicelles [5-7]. All three structures show a 19strand anti-parallel beta-barrel with the N-terminal domain (a partly alpha-helical strand of approximately 20 residues) inserted into, and interacting with residues of, the pore lumen. There can be no question that the 19-strand beta-barrel motif represents a common low-energy state of the protein. However, the motif disagrees with a prevailing model for VDAC having far fewer (13) transmembrane strands, based on results from planar bilayer experiments. Thus, the "biological relevance" of the atomic structures has been challenged [8]. In effect this is a debate between the validity of a structure determined using proteins extracted and refolded in detergent from bacterial inclusion bodies and a structure inferred from experiments using proteins purified in detergents and reconstituted into artificial lipid bilayers. Cryo-EM is helping to inform this debate by data obtained two decades ago about the structure of VDAC in its normal environment, the mitochondrial outer membrane, in the absence of detergent extraction and protein refolding or reconstitution [9]. The atomic structures of VDAC show excellent agreement with the projected density map of VDAC obtained by correlation averaging of EM images of ordered arrays of the 
channel in frozen-hydrated outer membranes isolated from mitochondria of the fungus Neurospora crassa. In addition to linking the 19-beta-strand motif to the structure of the mitochondrial pore in its native membrane environment, EM data indicate that the $\mathrm{N}$-terminal domain is mobile and accessible to antibodies at least some of the time. Thus, movement of the $\mathrm{N}$-terminal domain in and out of the pore lumen could be responsible for the observed changes in VDAC conductance.

2. The nature of outer-inner membrane contact sites

EM images of conventionally fixed and plastic-embedded mitochondria (isolated or in situ) suggest that the OM and IM come in frequent contact. The appearance of these "contact sites" has led to considerable speculation about their structure (e.g., regions of membrane fusion involving nonbilayer lipid phases), and function, including metabolite channeling involving VDAC in the OM and the adenine nucleotide carrier (ANT) in the IM [10]. Moreover, the phenomenon of a mitochondrial permeability transition that short circuits the mitochondrial IM potential has been attributed to interactions of VDAC, ANT and several other proteins at OM-IM contact sites. We are in the process of a careful examination of tomograms of isolated, frozen-hydrated mammalian and fungal mitochondria and of mitochondria in frozen-hydrated mammalian cells and tissue. The emerging conclusion is that sites of apparent contact between OM and IM are rare and there is no evidence of OM-IM fusion. In addition, in some tomograms, numerous 10-nm particles can be observed both in the space between the OM and IM and inside cristae, bridging adjacent IM surfaces. These inter-membrane particles may normally mediate "contact" between the OM and IM. They may be responsible for maintaining the remarkably constant spacing observed between OM-IM and IM-IM surfaces in frozen-hydrated mitochondria (but lost in some pathological states) and, at the same time, tether the IM to the OM and to itself, thereby stabilizing IM topology.

\section{References}

[1] D.C. Wallace, Annu. Rev. Biochem. 76 (2007) 781

[2] C.A. Mannella, Biochim. Biophys. Acta 1762 (2006) 3

[3] C.-H. Hsieh et al., J. Struct. Biol. 153 (2006) 1

[4] M. Marko et al., Nature Meth. 4 (2007) 215

[5] S. Hiller et al., Science 321 (2008) 1206

[6] R. Ujwal et al., Proc. Natl. Acad. Sci. USA 105 (2008) 17742

[7] M. Bayrhuber et al., Proc. Natl. Acad. Sci. USA 105 (2008) 15370

[8] M. Columbini, Trends Biochem. Sci. 34 (2009) 382

[9] X.W. Guo et al., J. Struct. Biol 114 (1995) 41

[10] D.G. Brdiczka, Biochim. Biophys. Acta 1762 (2006) 148

[11] Supported by NIH / NCRR Biomedical Research Technology Program grant P41 RR01219 and grants from NSF. 